\title{
EVALUACIÓN DEL SISTEMA DE ASESORÍA Y APOYO A FAMILIAS CON BEBÉS MÚLTIPLES EN LA CIUDAD DE QUITO
}

\author{
${ }^{1}$ Ana Cevallos Vacas, ${ }^{2}$ Alexandra Clavijo Loor \\ 1Universidad Tecnológica Equinoccial, Quito - Ecuador, cvac1025333@ute.edu.ec \\ 2Universidad Tecnológica Equinoccial, Quito - Ecuador, clma97902@ute.edu.ec
}

Recepción/Received: 23, 09, 2014

Aceptación/Accepted:01, 12, 2014

Publicado/Published: 12, 12, 2014

\begin{abstract}
Resumen
La presente investigación atiende la problemática de los embarazos múltiples y la orientación de las madres y padres frente a esta situación, la organización y el día a día con dos o más bebés que requieren de la asistencia de sus necesidades, sin que exista actualmente en Quito un programa psico-profiláctico dirigido específicamente para futuras familias con bebés múltiples. Realizamos entrevistas a profesionales médicos para conocer los cambios físicos y emocionales que las madres atraviesan desde el embarazo hasta el nacimiento de sus hijos o hijas, y trabajamos con grupos focales para conocer las diferentes experiencias de madres con bebés múltiples y temas que les hubiera gustado conocer antes del nacimiento de sus criaturas, ya que son estos aspectos los que se tratarían en las charlas y asesorías a padres y madres que atraviesan por estas circunstancias.. Tanto los profesionales como las madres de bebés múltiples recomiendan la asistencia a estas charlas a nuevas familias para disminuir el estrés y preocupación causado por el embarazo múltiple.
\end{abstract}

\section{Palabras clave}

Cuidados de bebés múltiples, embarazos múltiples, sistema de asesorías.

\begin{abstract}
The present investigation is aimed at the orientation of parents who experience multiple pregnancies and births. Such orientation focuses on the organization demanded for everyday work with two or more babies that require assistance in their care and necessities. Currently, there is no psychoprophylactic program in Quito directed specifically for future families of multiple babies. Therefore, professional doctors were interviewed to know about the physical and emotional changes that the mother goes through from the pregnancy until the babies are born; as well as interviews to support groups in order to learn the different experiences of multiple birth mothers and discuss issues that these mothers would have been willing to know before their babies were born. Chats and counseling meetings would be a good opportunity for parents of multiple pregnancies to share and discuss their concerns. Doctors, as well as parents, recommend that new families attend these chats to lower stress and relieve worries caused by these kinds of pregnancies.
\end{abstract}

\section{Keywords}

Counselling system, multiple baby care, multiple pregnancies. 
adulta, señala que:

\section{INTRODUCCIÓN}

La confirmación de que son dos o más bebés los que vienen en camino, sin duda acarrea preocupaciones y estrés para las madres y padres ante la llegada de los niños y niñas que requerirán de mayor organización y control en el hogar. Varias son las preocupaciones que los padres y madres de bebés múltiples deben asumir y que son diferencias trascendentales en comparación con un embarazo único; desde la lactancia hasta la manera de dormir son inquietudes constantes al no tener ninguna experiencia previa en embarazos múltiples. Es en este contexto que, como maestrante y madre de bebés múltiples, decidí investigar y ayudar a las familias que se encuentran en esta situación. El presente estudio ha sido realizado conjuntamente con mi directora de tesis, como trabajo final de grado para la Maestría en Educación Especial.

Entre la bibliografía revisada para la presente investigación encontramos dos libros de apoyo importante: 9 meses con combo de gemelos, escrito por Carley Roney (2013) y El gran libro de los gemelos, desde el embarazo múltiple hasta la edad adulta, escrito por Coks Feenstra (2011), que nos sirvieron de guía y orientaron nuestro trabajo.

La valoración del sistema de charlas y asesorías a padres y madres de bebés múltiples en cursos de profilaxis o en hospitales y clínicas de la ciudad de Quito fue la principal preocupación de este estudio, así como los diferentes aspectos que se deberían tratar en estas reuniones. Adicionalmente, nos interesaba conocer si a las familias múltiples les hubiera servido el contar con una capacitación previa al nacimiento de sus bebés y si las recomendarían a futuras familias que se encuentren en similares situaciones. Estos fueron nuestros principales objetivos para la presente investigación.

\section{DESARROLLO DEL ARTÍCULO}

Los partos múltiples en la actualidad se han multiplicado significativamente. Feenstra (2011) en su libro El gran libro de los gemelos, desde el embarazo múltiple hasta la edad
Los partos múltiples han aumentado espectacularmente en los últimos veinte años. Espontáneamente uno de cada 80 embarazos es gemelar; uno de cada 6000 es de trillizos, y uno de cada 500000 es de cuatrillizos. Estas cifras son aproximadas y varían dependiendo de la zona del globo; en algunas regiones de África, como Nigeria, casi uno de cada 40 embarazos es de gemelos, mientras que los embarazos gemelares en Asia son excepcionales. (p.1).

Varios son los motivos por los que una mujer puede quedar embarazada de bebés múltiples; en la actualidad la incursión de las mujeres al mundo laboral ha provocado que su maternidad sea postergada a una edad mayor; es así como el promedio actual de su concepción es de 34 - 35 años de edad, mientras que hace sólo dos décadas atrás el promedio de la maternidad era los veinte años. Como se sabe, se es mucho más fértil en esta edad. Factores adicionales como el consumo de tabaco y alcohol, así como substancias tóxicas, son otros motivos por los que las mujeres no pueden concebir de forma natural.

El incremento en los tratamientos de fertilidad refleja el aumento de bebés múltiples. En la actualidad es mucho más común el ver a padres y madres con dos o más bebés caminando por un centro comercial.

Los diferentes avances tecnológicos han hecho que los tratamientos sean cada vez más eficientes. En Ecuador, los partos múltiples han crecido significativamente: en el 2009 se registraron 739 casos de partos gemelares y once partos múltiples sin especificar el número de bebés nacidos, versus 768 partos gemelares registrados en el 2011 y doce partos múltiples restantes, lo que nos da un crecimiento del 3,92\%, y es la Provincia de Pichincha la que registra un mayor número de partos múltiples. En el 2012 las estadísticas revelan que fueron 754 partos gemelares y 17 partos múltiples sin determinar el número de bebés nacidos (Ministerio de Salud Pública, 2009 - 2012).

La presente investigación responde a nuestro posicionamiento en concordancia con el enfoque cualitativo, prioritariamente presente en la redacción del informe, la concepción para la generación de información y el tratamiento de la misma, la compresión de la investigación social y sus aportes al desarrollo humano. También 
hemos colocado algunos rasgos cuantitativos, como se puede observar en la presentación mínima de cantidades y porcentajes, que nos han servido para caracterizar la población de estudio, así como algunos aspectos de lenguaje propios de la lógica cuantitativa.

Nuestra investigación inició con la visita a cinco principales hospitales de la ciudad de Quito, considerando tres estratos sociales: alto, medio y bajo, para conocer si en ellos se dictaban charlas o asesorías a padres y madres de bebés múltiples. Adicionalmente, visitamos dos centros de estimulación temprana y profilaxis, correspondientes a los estratos sociales alto y medio, pues en la sociedad ecuatoriana, cuando las parejas conciben a un hijo o hija normalmente visitan estos lugares para recibir cursos profilácticos. Consideramos importante incluirlos, puesto que podían ser los lugares ideales para charlas específicas a futuros padres y madres de bebés múltiples.

Entrevistamos a tres reconocidos doctores especialistas en embarazos múltiples a nivel local: un ginecólogo, un pediatra y una psicóloga, para conocer los diferentes cambios físicos y emocionales que tienen las madres y padres cuando se les confirma la llegada de dos o más bebés, así como precauciones que son importantes para lograr que el embarazo llegue a término y, tanto la madre como las criaturas, no tengan ningún tipo de inconveniente.

Realizamos dos grupos focales con madres de bebés múltiples: un grupo compuesto por cinco madres con bebés múltiples menores a un año de edad, y un grupo compuesto por cuatro madres de bebés múltiples mayores a un año; quienes aportaron significativamente a esta investigación con la finalidad de esclarecer interrogantes como la siguiente: ¿Cuáles fueron sus principales inquietudes y experiencias durante su embarazo, y una vez que llegaron a casa con sus bebés? Sus respuestas nos ayudaron a comprender las diferencias con padres y madres de bebés únicos, y a especificar los problemas más frecuentes que atravesamos las familias de bebés múltiples.

Previamente a la realización de la ficha de observación de campo, entrevistas a profesionales y grupos focales, las preguntas fueron validadas por personas especialistas en el medio (doctores, madres con experiencia en bebés múltiples y profesionales en investigación), con la finalidad de asegurar que las mismas se ajustaran a la realidad de las personas entrevistadas y los resultados pudieran ser reales y transcendentes para la investigación. Se utilizó grabación de voz y video como soporte técnico para archivar la información que posteriormente fue analizada.

Adicionalmente, utilizamos fichas de observación, anecdotarios y diario de registro de cuatro familias múltiples, en los que se especificaba la rutina de cada uno de sus bebés.

Estos instrumentos fueron utilizados con la finalidad de llevar un registro de las actividades durante el tiempo de la investigación, para no perder detalle alguno.

\section{RESULTADOS Y DISCUSIÓN}

De los hospitales y clínicas visitadas, ninguno de ellos incluía charlas a padres y madres de bebés múltiples; solo en el Hospital Gineco-Obstétrico Isidro Ayora nos comunicaron que el médico encargado de recibir a los bebés el momento en que su madre daba a luz, debía ser el encargado de capacitar a las madres y a su pareja antes de que les dieran el alta; es decir, en un tiempo de tres días debía organizarse para tratar de hacer diferentes recomendaciones sobre el cuidado y crianza de los recién nacidos. Es importante mencionar que ninguno de los doctores de dicho lugar tenía experiencia en neonatología de bebés múltiples.

En cuanto a los centros de estimulación temprana y profilaxis, si bien dictaban constantemente charlas de asesoría a futuros padres y madres de bebés, se enfocaban exclusivamente a bebés únicos y no múltiples. Nos comentaron que tuvieron madres embarazadas de dos o más bebés en sus charlas, pero que no supieron responder temas específicos sobre la crianza de bebés múltiples e incluso llegaron a ofrecernos trabajo en caso de tener madres y padres interesados, pues la necesidad estaba presente.

De igual forma, tanto los doctores entrevistados como las madres que participaron en los grupos focales, no conocían de algún lugar a donde recurrir específicamente para temas de bebés múltiples.

Esta investigación nos da como resultado que en Ecuador, específicamente en Quito, pese a que los embarazos múltiples se han incrementado desde los últimos cinco años, no existe ningún tipo de charlas o asesorías a futuros 
padres y madres para prepararse antes de la llegada de sus bebés. Este incremento nos confirmó tanto el Dr. Marcelo Flores, ginecólogo, especialista en fertilidad; así como el Dr. Código 001, pediatra - neonatólogo experto en bebés múltiples, y que para fines de esta investigación prefirió no ser identificado con su nombre real en las entrevistas realizadas.

A pesar de que cada vez resulta más habitual ver a madres y padres con bebés múltiples, el embarazo múltiple es considerado de alto riesgo tanto para la madre como para sus hijos e hijas; el cuerpo humano está diseñado para la concepción de un solo bebé y cuando existen más vidas siempre existirán más riesgos, en especial la posibilidad de que las criaturas nazcan de forma prematura.

Adicionalmente a los cuidados que la madre deberá tener durante el embarazo, el impacto psicológico que soportan los padres y madres cuando se les confirma la noticia de que será más de un bebé, es un hecho que deberá asimilarse y aceptar lo más pronto posible para no interferir en el bienestar y desarrollo de las criaturas. La Dra. Ma. Judith Almeida explicó este proceso:

Claro, es impactante el hecho de saber que uno tiene más de un embarazo; desde el principio, uno está preparado para ser madre de uno; uno dice me quedo embarazada y hablas de mi bebé, nunca hablas de mis bebés; entonces todo va por el deseo; el embarazo viene por el deseo, es cultural que una se quede embarazada de un hijo o de una hija. De pronto te dicen, van a ser dos, o van a ser tres, ya es un impacto emocional porque tú dices: yo soy una sola persona, ¿cómo voy a hacer caso a más de un hijo? En caso de ser primeriza, impacta el hecho y en caso de no ser primeriza, ya cuentas con un hijo anterior; entonces tú vas, emocionalmente multiplicándote y piensas: voy a necesitar más tiempo, más dinero, voy a necesitar más espacio. Entonces empiezas a hacerte un conflicto. (Dra. Almeida, 2014).
Sin embargo, si bien los primeros seis meses de vida son considerados como los más difíciles hasta organizarse y adaptarse al nuevo estilo de vida, los padres y madres de bebés múltiples llegamos a realizar un buen trabajo. El Dr. Código 001, pediatra - neonatólogo nos compartió su experiencia con padres y madres múltiples:

Otras parejas que tienen gemelos, caramba, hacen un mejor trabajo con dos que con uno, la mamá se estresa menos con dos que con uno, por el tema que te decía: maduran, se vuelven más responsables, se ven ante una situación que tal vez no estaba prevista, no se imaginaron que iba a ser así y psicológicamente cambian, se lo proponen y lo logran. (Dr. Código 001, 2014).

La realidad de varios bebés es aceptada por las familias de bebés múltiples de distintas maneras. Para algunas personas es un proceso normal por haber tenido experiencia previa en sus familias, pero también están aquellas familias primerizas que carecen de conocimiento y experiencia sobre el desarrollo de los bebés. A estas familias les cuesta más la adaptación.

De los dos grupos focales realizados, el 90\% de las madres participantes afirmaron que sí necesitaron de ayuda adicional durante los primeros días de llegados los recién nacidos. Esta ayuda podía ser de diferentes miembros de su familia o de enfermeras capacitadas, según cada caso en particular.

Ninguna de las madres que colaboró en los grupos focales, encontró un lugar donde poder compartir sus inquietudes sobre la llegada de los bebés.

Al 88\% de las mismas les preocupaba mayormente el tema de la lactancia múltiple y la organización en el hogar; y el conocer cómo establecer la rutina diaria con dos o más bebés, así como el conocer si los bebés múltiples lloran al mismo tiempo.

El tema de alergias y sus tratamientos preocupaba al $44 \%$ de los grupos focales, mientras que temas como las horas 
de sueño y el baño preocupaba al 33\%.

Las charlas y asesoría a padres y madres de bebés múltiples para prepararse antes de la llegada de los bebés son aceptadas en todas las madres de nuestro grupo de estudio; grupos en donde puedan compartir sus diferentes inquietudes y conocer a madres y padres que se encuentren en similares situaciones podrían ayudar a sobrellevar el día a día con dos o más bebés. Los temas especificados anteriormente, que preocupaban a las madres, serían los que se incluyan en las charlas de asesoría y apoyo, además de temas sobre el embarazo, cuidado y precauciones que deberán tener las madres durante su periodo gestacional para evitar partos prematuros.

Las charlas y asesorías a padres y madres de bebés múltiples una vez que ya han nacido los bebés, son aceptadas por el 95\% de las madres que participaron en los grupos focales, siempre y cuando se topen temas específicos como disciplina, técnicas para la hora de dormir y tips para mejorar la alimentación de sus bebés, que estuvieron entre las principales sugerencias. El 11\% de las madres mencionó la importancia de establecer límites con los demás miembros de la familia para evitar problemas con la pareja, que incluso podrían llegar al divorcio.

En todas las estrategias de investigación utilizadas (entrevistas a tres (3) doctores: ginecólogo, psicólogo y pediatra, así como en los dos grupos focales a madres múltiples), se reconoció la importancia de contar con charlas y asesorías a futuros padres y madres de bebés múltiples. La alimentación y cuidado diario de los recién nacidos, son algunas de las muchas diferencias existentes entre niñas y niños únicos a múltiples. No es lo mismo tener un solo bebé a dos o más; la organización y el control son factores primordiales en familias múltiples.

El compartir experiencias, sin duda enriqueció nuestro trabajo; las madres de bebés múltiples estuvieron totalmente abiertas a contar sus experiencias, tanto sus aciertos como desaciertos y a encontrar diferentes opciones para sus propios problemas.

Las charlas de asesoría y apoyo que proponemos en este trabajo constituirían un espacio para que todas las madres de bebés múltiples pudieran compartir experiencias y se sintieran apoyadas entre ellas, de tal modo que, en conjunto, se buscaran soluciones al día a día en esta hermosa experiencia.

\section{CONCLUSIONES Y RECOMENDACIONES}

- En la actualidad, en Quito no existe ninguna persona o institución que dicte charlas específicas a futuros padres y madres de bebés múltiples.

- De la investigación realizada, a nivel internacional, sabemos que existen algunos países como Estados Unidos o Argentina, que ofrecen charlas dirigidas específicamente para padres y madres de bebés múltiples y en donde, adicionalmente, se crean foros instantáneos para que las madres y padres puedan acceder y solucionar sus inquietudes de forma inmediata. En este sentido, en Ecuador, en especial en Quito, que es la cuidad que registra un mayor número de partos múltiples, podríamos también realizar este tipo de interacciones como ayuda a todas estas familias.

- Algunos de los temas a incluir en caso de existir charlas de asesoría y apoyo a padres y madres de bebés múltiples para prepararse antes y después de la llegada de los bebés serían los siguientes: cuidados y precauciones, lactancia múltiple, rutina diaria, alimentación y alergias o enfermedades.

- Con respecto al sistema de asesoría y apoyo a padres y madres de bebés múltiples, nuestra investigación revela que es insuficiente en la ciudad de Quito; y son sus pares el único apoyo posible a nivel informal para estas familias,, con base en la experiencia y comentarios de amistades y conocidos que han pasado por las mismas circunstancias, sin un sustento científico o profesional.

- Las charlas de asesoría y apoyo son de gran importancia para padres y madres de bebés múltiples, las mismas que deben ayudar durante el proceso de adaptación desde el embarazo hasta el nacimiento de sus bebés. El contar con estas charlas disminuiría la ansiedad ocasionada por la confirmación de que vienen dos o más bebés, así como el sentirse apoyados por personas que comparten la misma experiencia 


\section{AGRADECIMIENTOS}

Nuestro sincero agradecimiento a todas las personas que fueron parte de la investigación, profesionales en diferentes áreas del conocimiento, madres y padres de bebés múltiples, personal de las instituciones de salud. A ellos les reconocemos sus importantes aportes como voces de este trabajo de investigación y con quienes compartimos los méritos de esta publicación.

\section{REFERENCIAS BIBLIOGRÁFICAS}

Feenstra, C. (2011). El gran libro de los Gemelos, desde el embarazo múltiples hasta la edad adulta. Barcelona: Medici.

Ministerio de Salud Pública. Coordinación General de Planificación. Dirección General de Información, Seguimiento y Control de Gestión. Número de Partos Gemelares y Partos Otros por Provincia. Producción de Establecimientos de Salud. 2009 2012.

Prindle, P. (22 de Mayo de 2013). Before you Deliver Multiples: Childbirth Preparation Classes for Parents ofTwins or More. Obtenido de http://multiples.about. com/od/pregnancy/a/twinbirthclass.htm.

Roney, C. (2012). 9 meses con combo de gemelos: Todo cuanto hay que saber para sobrevivir a tu embarazo múltiple. Bogota: Editorial llustrados Grijalbo/Lumen 